\section{Pneumatosis Cystoides Intestinalis, Is the Emergency Surgery Inevitable: Case Report}

\section{Abstract}

Pneumatosis cystoides intestinalis is a rare clinical phenomenon in which there is presence of gas filled cysts in the gut wall. Its exact cause is not known but this condition is commonly associated with smoking and other factors leading to raised intra-abdominal pressure. Sometimes these gasses filled cysts may rupture on their own or secondary to some surgical intervention leading to presence of free gas in the abdomen or retroperitoneum. We present here a case report of the patient who presented to the emergency room with pain abdomen and a large amount of free gas in the abdomen seen on erect X-ray of the abdomen. Here comes the challenge to the surgeon, whether to operate or not. Contrast Enhanced Computerised Tomography (CECT) scan of the abdomen is a better modality for diagnosing Pneumatosis cystoides intestinalis ( $\mathrm{PCl}$ ) and it can prevent unnecessary surgeries. However, the management of this condition is mainly nonsurgical, and oxygen forms the mainstay of therapy.

Keywords: Pneumoperitoneum; Pneumoretroperitoneum; Pneumobilia; Oxygen therapy

Received: August 29, 2017; Accepted: October 27, 2017; Published: November 04, 2017

\section{Sharma R, Chaudhary R*, Bharti R, Verma A, Verma RK and Bodh Negi TD}

\author{
Department of Surgery, Dr. Rajendra Prasad \\ Government Medical College Kangra, \\ Himachal Pradesh, India
}

\section{*Corresponding author: Chaudhary R \\ topgun.chaudhary@gmail.com}

Department of Surgery, Dr. Rajendra Prasad Government Medical College Kangra, Himachal Pradesh, India.

\section{Tel: 01892267115}

Citation: Sharma R, Chaudhary R, Bharti $R$, Verma A, Verma RK, et al. (2017) Pneumatosis Cystoides Intestinalis, Is the Emergency Surgery Inevitable: Case Report. Transl Biomed. Vol.8 No.4:136

\section{Introduction}

Pneumatosis Cystoides Intestinalis (PCI) is a rare condition, which is otherwise a harmless collection of air within the gut wall giving rise to multiple air-filled cysts, but when these cysts rupture they may produce pneumoperitoneum and hence complicate the clinical scenario. The patients usually present to emergency room (ER) with some other ailments but the abdominal X-rays reveal free gas under diaphragm in about two thirds of the patients. They usually have a history of chronic respiratory disorders like bronchitis and asthma, malabsorption syndrome, connective tissue disorders or intake of substrates which remain unabsorbed inside the gut like lactulose or sorbitol [1]. Smoking is a commonly associated factor as described by Hughes et al. in their case series of five cases where four patients were smokers. In children, this condition is seen with necrotising enterocolitis. Although $\mathrm{PCl}$ is not a premalignant condition, but it has been seen in association with carcinoma stomach, lymphosarcoma and widespread carcinomatosis of the abdomen [2]. These patients showing pneumoperitoneum or pneumoretroperitoneum are a management dilemma. CECT abdomen is the definitive method of diagnosing this condition and it can save undue surgeries for a condition whose treatment is otherwise conservative.

\section{Case Report}

A 70-year-old male patient was referred to our medical college from a peripheral health institution with the history of pain upper abdomen for one day and an erect X-ray abdomen showing large amount of free gas under diaphragm (Figure 1). The patient was a smoker for last 50 years and had history of taking proton pump inhibitors for pain abdomen, off and on in the past. There was no history suggestive of any connective tissue disorder or inflammatory condition in the past. Blood pressure was $116 / 90$ $\mathrm{mm}$ (millimetres) of mercury. Pulse rate was 90 beats per minute. Abdominal examination revealed mild distension with tenderness in the epigastrium and right hypochondrium region. Blood examination revealed raised leucocyte count (14000 per cubic millimetre). Other haematological and biochemical parameters were within normal limits. On the basis of history, clinical examination and erect X-ray abdomen showing free gas under diaphragm, the possibility of perforated duodenal ulcer was kept. Explorative laparotomy was performed on the patient. On opening the abdominal cavity, we were amazed to see whole of the gut to be studded with multiple air-filled cysts of variable size. No hollow viscus perforation was seen. Appendix was also normal (Figure 2). There was no free fluid in the abdominal cavity. 


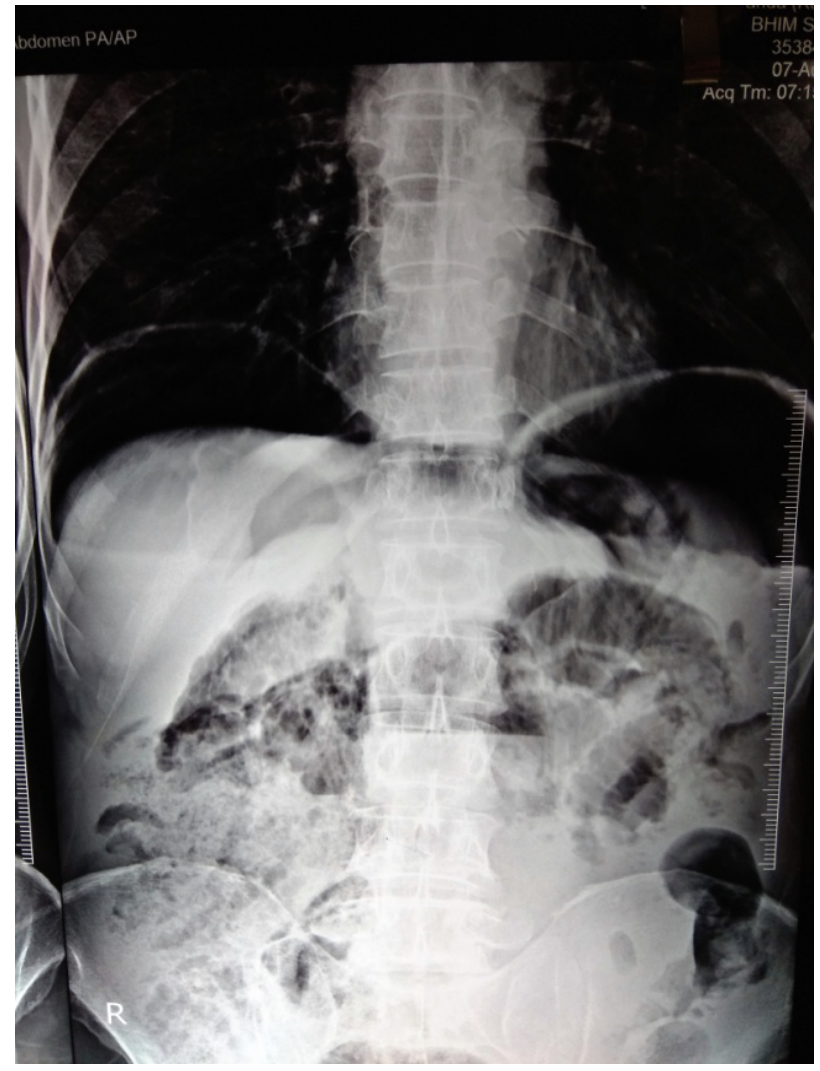

Figure 1 Showing free gas under diaphragm and multiple airfilled cysts in the small intestine.

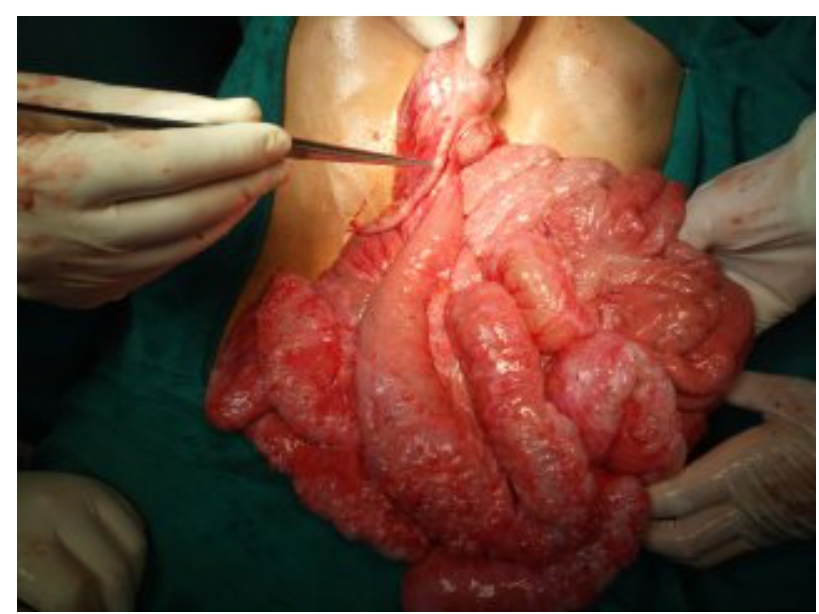

Figure 2 Showing air filled cysts of the small intestine and a normal appendix.

Abdomen was closed after keeping a drain in the pelvis. Patient was given high flow oxygen in the ward. Drain was removed after 24 hours and patient discharged home after 04 days. On re-examining the X-rays, we could make out multiple air-filled spaces in the small intestine. The patient has recovered well and is still in follow up after six months.

\section{Discussion}

Presence of air inside the gut wall forming the gas filled pseudocysts is known as Pneumatosis Cystoides Intestinalis. Although this condition was described first in 1730, by Duvernoi but it is still a little less known and often misunderstood condition [1]. The exact cause of this condition is not known but it is mostly secondary (85\%) to the other illnesses like chronic obstructive airway disease. In about $15 \%$ cases, the cause is not known [3]. Small intestine is the most frequently involved part of the intestine which is generally seen secondary to other causes. Sigmoid colon is the most commonly involved part of the large intestine and is usually seen in primary type [4]. Gas filled cysts are not only confined to the gut but are also seen in omentum, mesentery, stomach, oesophagus, peritoneum, retroperitoneum and portal venous system. Rarely air-filled cysts may be seen in the lymph nodes $[1,5]$. The clinical presentation of this disease poses a challenge to the surgeon. This condition is more common in males aged between 30 to 50 years. The patients commonly present with an underlying illness and it is only during the investigations that they are detected with $\mathrm{PCl}$ [6]. Hughes et al. found it to be associated with smoking and chronic obstructive airway disease [2]. Etiopathogenesis of this condition is a mystery too even after more than two hundred and fifty years of its first description. There are many theories associated with its genesis. A theory says that raised intra-abdominal pressure and the breach of mucosal integrity lead to the formation of such air-filled cysts. Sometimes chronic coughing can force the air through diaphragm into the abdominal cavity where it diffuses through the blood vessels and reaches the gut wall through the mesentery, but recent studies believe it to be due to a changing intra-abdominal pressure. In connective tissue disorders and inflammatory conditions, invasive microorganisms can breach the mucosal barrier. Over production of hydrogen, either due to bacteria or unabsorbable substrate like lactulose inside the lumen of the intestine can force it through the mucosal breaches to form the air-filled cysts [1]. The content of these cysts has been analysed and Mujahed et al. found $89 \%$ of nitrogen in these cysts. Other gases seen in the cysts are hydrogen, oxygen and carbon dioxide [7]. These cysts may rupture and can produce pneumoperitoneum or pneumoretroperitoneum in about $30 \%$ of the cases. This gas can be seen on $X$ - ray examination of the abdomen in about $66 \%$ of cases [8]. This produces the dilemma like in our case. X-ray of the abdomen revealing large amount of free gas under both the domes of diaphragm with mild tenderness in the right hypochondrium prompted us to go for exploratory laparotomy. These patients may present with a wide range of clinical features ranging from diarrhoea, rectal bleeding, constipation, and weight loss secondary to malabsorption. Sometimes they may present with intussusception, volvulus or complete intestinal obstruction [4]. On digital rectal examination, the submucosal cysts may give a cushion like feel and on sigmoidoscopic or colonoscopic examination they may be misinterpreted as polyps. They may disappear on needle puncture for biopsy as it happened with Cyrany [1]. People 
have even utilised ultrasonography, barium studies, Magnetic Resonance Imaging (MRI) and even laparoscopy for diagnosing $\mathrm{PCl}$. Stable patients with pneumoperitoneum should be subjected to Contrast Enhanced Computerised Tomography (CECT) scan of the abdomen. It is the investigation of choice which will show air filled cystic spaces within the bowel wall, retro peritoneum and portal circulation. It will also tell the status of lungs in case of chronic smokers where this condition is most commonly seen [5]. Treatment of this condition is mainly conservative. Treating the underlying condition will lead to resolution of this disease. Medical management requires high flow oxygen, dietary modifications and sometimes antibiotics like metronidazole for a long duration. Oxygen diffuses into the cysts and forces the other gases out into the blood. Hyperbaric oxygen at 2.5 atmosphere pressure for two hours per day can reduce the duration of oxygen therapy [9]. Endoscopic treatments like polypectomy should be

\section{References}

1 Cyrany J, Kopaaova M, Hornychova H, Ryska A, Rejchrt S, et al. (2005) Pneumatosis cystoides intestinalis. A case report and review of the literature. Folia Gastroenterol Hepatol 3: 68-73.

2 Hughes DT, Gordon KC, Swann JC, Bolt GL (1966) Pneumatosis cystoides intestinalis. Gut 7: 553-557.

3 Boerner RM, Fried DB, Warshauer DM, Isaacs K (1996) Pneumatosis intestinalis. Two case reports and a retrospective review of the literature from 1985 to 1995. Dig Dis Sci 41: 2272-2285.

4 Davila AD, Willenbucher RF (1998) Pneumatosis cystoides intestinalis. In: Sleisenger and Fordtran's Gastrointestinal and Liver Diseases. Feldman M, Scharschmidt BF, Sleisenger MH (eds), (6th edn). Philadelphia: WB Saunders, USA. pp. 1979-1982. avoided as it can lead to perforation and peritonitis. Surgical management should be considered whenever a patient presents with features of ischemic gut, intestinal obstruction, perforation, sepsis or features of peritonitis [1].

\section{Conclusion}

Not every patient having free gas under diaphragm is a candidate for surgery. Rather they should be investigated for underlying causes whenever the condition permits. Most of us will rarely see this condition more than once in our surgery careers so high index of suspicion is required for diagnosing this condition. CECT abdomen should be utilised more often than normal in patients having pneumoperitoneum without any features of peritonitis. Oxygen therapy is the main stay of treatment for $\mathrm{PCl}$ with surgery being only rarely ever required.

5 Rahim H, Khan M, Hudgins J, Lee K, Du L, et al. (2013) Gastrointestinal sarcoidosis associated with Pneumatosis cystoides intestinalis. World J Gastroenterol. 19: 1135-1139.

6 Goodman RA, Riley TR (2001) 3rd. Lactulose-induced Pneumatosis intestinalis and pneumoperitoneum. Dig Dis Sci 46: 2549-2553.

7 Mujahed Z, Evans JA (1958) Gas cysts of the intestine. Surg Gynec Obstet 107: 151-160.

8 Myre LE, Pinon S, Forman WB (2004) Causes of benign asymptomatic pneumoperitoneum. Am Fam Physician 69: 29-32.

9 Azzaroli F, Turco L, Ceroni L, Galloni SS, Buonfiglioli F, et al. (2011) Pneumatosis cystoides intestinalis. World J Gastroenterol 17: 49324936. 